particles (UFP) in the environment, particularly in urbanised areas. In the last years, there is an increasing use of biomassderived fuels because they are a renewable source of energy that may mitigate climate change through the reduction of net $\mathrm{CO}_{2}$ with respect to conventional fossil fuels. Although there is a general agreement on biofuels ability to reduce conventional pollutants, new and potentially harmful pollutants can be formed during biofuel combustion. In particular, the emission of sub-10nm particles is strongly increased with respect to that of larger soot particles.

Methods Organic sub-10nm particles are separated from larger sizes particulate matter by collection in water suspension for toxicological and inflammatory tests. After exposure to sub$10 \mathrm{~nm}$ particles, the effects on proliferation, apoptosis and secretion of cytokines, chemokines and growth factors networks production is analysed in immortalised non-tumorigenic human dermal keratinocyte cell line (HaCaT) and human alveolar epithelial-like cells (A549).

Results Nanoparticles exert different cytotoxic effects in the two cell lines, suggesting that the dermal way of exposure is more sensitive than the inhalant way. These differences are most evident in the secretion of pro-inflammatory, angiogenic and proliferative cytokines and chemokines whose expression is more finely modulated in HaCaT cells compared to A-549 cells.

Conclusion Considering the size of these particles, it is important to promote the culture of prevention also for the dermal way in particularly exposed workers.

\section{8d ALTERATION OF IMMUNE CELLS IN SILICOSIS: ROLES IN DEVELOPMENT OF AUTOIMMUNITY AND LUNG FIBROSIS}

${ }^{1} \mathrm{~T}$ Otsuki, ${ }^{2} \mathrm{H}$ Hayashi, ${ }^{1} \mathrm{~S}$ Lee, ${ }^{1,3} \mathrm{H}$ Matsuzaki, ${ }^{1} \mathrm{~N}$ Kumagai-Takei, ${ }^{1} \mathrm{~K}$ Yoshitome, ${ }^{1} \mathrm{Y}$ Nishimura, ${ }^{2} \mathrm{~W}$ Fujimoto. ${ }^{1}$ Department of Hygiene, Kawasaki Medical School; ${ }^{2}$ Department of Dermatplogy, Kawasaki Medical School; ${ }^{3}$ Department of Life Science, Faculty of Life and Environmental Science, Prefectural University of Hiroshima, Shobara, Japan

\subsection{6/oemed-2018-ICOHabstracts.141}

In addition to lung fibrosis, silicosis (SIL) patients often suffer from complicated autoimmune disorders such as rheumatoid arthritis, systemic sclerosis and anti-neutrophil cytoplasmic antigen-related vasculitis/nephritis. Thus, chronic and recurrent exposure to silica particles located in the lung and lymph nodes can result in alterations in the function of immune cells, which can lead to the dysregulation of autoimmunity in addition to the development of lung fibrosis. Regarding B cells which produce various antibodies, in SIL many autoantibodies are often detected in autoimmune diseases, and specifically autoantibodies against apoptosis-related molecules. Responder $\mathrm{T}$ helper ( $\mathrm{rTh}$ ) cells which respond to foreign and auto-antigens have been reported to survive longer and have apoptosis inhibited. Additionally, regulatory $\mathrm{T}$ (Treg) cells seem to proceed to early apoptosis. This imbalance between rTh and Treg cells may make SIL patients prone to autoimmune disorders. Although the role of dendritic cells (DCs) including alveolar macrophages and T helper 17 (Th17) cells in the dysregulation of immune tolerance in SIL remains poorly understood, these cells play a role in pulmonary inflammation and the development of fibrosis via specific receptor and signalling molecules. Further studies are required to delineate the roles of DCs and Th17 cells in the disturbance of autoimmunity found in SIL, and investigation of the immunological alterations that lead to autoimmune dysregulation may assist in the recognition, prevention, and treatment of complicated autoimmune diseases found in SIL.

\section{ALLERGIES IN THE WORKPLACE}

${ }^{1}$ Claudia Petrarca*, ${ }^{2}$ Takemi Otsuki* ${ }^{1}$ O.U. Allergy and Immunotoxicology and Occupational Biorepository Centre of Excellence on Ageing (Ce.S.I.) Via Luigi Polacchi, Chieti, Italy; ' ${ }^{2}$ Department of Hygiene, Kawasaki Medical School, Kurashiki, Japan

\subsection{6/oemed-2018-ICOHabstracts. 142}

Aim of the session To discuss occupational allergies in basic mechanisms, testing and newer clinical topics

${ }^{1}$ Dr. A. Fourie, ${ }^{2}$ Dr. L. Wengenroth, ${ }^{3}$ Dr. E. Kuroda, ${ }^{4}$ Dr. M. Di Gioacchino

${ }^{1}$ National Institute for Occupational Health, National Health Laboratory Services, Johannesburg, South Africa

${ }^{2}$ Institute and Outpatient Clinic for Occupational, Social and Environmental Medicine, Munich, Germany

${ }^{3}$ Laboratory of Adjuvant Innovation, Centre for Vaccine and Adjuvant Research (CVAR), National Institutes of Biomedical Innovation, Health and Nutrition, Osaka, Japan

${ }^{4}$ Unit of Immunotoxicology and Allergy, CeSI-MET, G. d'Annunzio University, Chieti, Italy

\section{$1707 a$ TESTING FOR ALLERGY TO CHEMICAL PRESERVATIVES IN OCCUPATIONAL SETTINGS}

${ }^{1,2}$ A Fourie*, ${ }^{1,2} \mathrm{~T}$ Singh. ${ }^{1}$ National Institute for Occupational Health, National Health Laboratory Services, Johannesburg, South Africa; ${ }^{2}$ Department of Clinical Microbiology and Infectious Diseases, University of Witwatersrand, Johannesburg, South Africa

\subsection{6/oemed-2018-ICOHabstracts. 143}

Introduction Methylchloroisothiazolinone/Methylisothiazolinone (MCI/MI) and methylisothiazolinone (MI) are chemical preservatives found in cosmetics, industrial and household products. There is a reported epidemic of allergic reactions to these substances in several countries (e.g. increases of $4.1 \%$ per annum over 16 years in England). Workers that come into contact with the agents may develop occupational contact dermatitis. Therefore, detecting these allergens is important to better manage workers' skin condition and exposure. The aim of this study was to determine the prevalence of contact dermatitis to $\mathrm{MCI} / \mathrm{MI}$ and $\mathrm{MI}$ before and after changes in allergen testing was introduced.

Methods A retrospective assessment of workers referred to the NIOH Dermatology Clinic between 2006 and 2017 was conducted. Workers with work-related dermatitis were patch tested for sensitisation to MCI/MI and/or MI using the European baseline series (Chemotechnique). Frequencies of sensitisation to the allergens (MCI/MI and $\mathrm{MI}$ ) were calculated using Microsoft Excel.

Results A total of 583 occupational referrals were seen and 413 were patch tested to determine a possible allergic aetiology for the dermatitis. Sixteen patients (3.9\%) were positive to either one or both allergens (MCI/MI and MI) during the period January 2006 to June 2017. The testing concentration of the MCI/MI was doubled in 2014 and MI was introduced in 2015 . More cases were identified $(6 / 86 ; 7 \%)$ after changes were introduced post 2014 compared to previous allergen concentrations (10/327; 3.1\%). The workers identified with sensitisation to the chemicals were from occupations such as 
beauticians, cleaners, a millwright, a waitress and several workers that either decanted or mixed the chemicals.

Discussion $\mathrm{MCI} / \mathrm{MI}$ and $\mathrm{MI}$ are important occupational allergens and should be tested using the correct concentration to avoid misdiagnosing cases. The importance of keeping abreast with what is happening in the field was highlighted by the changes implemented which improved the detection of these allergens.

\section{7b FROM WORKPLACE TO HOME ENVIRONMENT: SPREADING OF MOUSE ALLERGENS BY LABORATORY ANIMAL WORKERS}

H Kube, L Wengenroth*, J Gerlich, G Dietrich-Gümperlein, R Schierl, D Nowak, K Radon. Institute and Outpatient Clinic for Occupational, Social and Environmental Medicine, Munich, Germany

\subsection{6/oemed-2018-ICOHabstracts. 144}

Introduction Laboratory animal workers (LAW) working with mice are exposed to mouse allergens (MA). Exposure to MA can lead to occupational allergies and asthma. If MA are spread to home environments, the longer duration of exposure might increase the risk for allergic symptoms. Little is known about the spreading of MA. This study aimed to assess:

- whether spreading of MA from workplace to home environment takes place,

- which factors increase spreading of MA.

Methods In a cross-sectional study we took dust samples from the homes of 107 LAW and 13 controls. From 90 LAW we took additional dust samples from their working place. Samples were analysed using mus $m 1$ ELISA kits. Through a questionnaire we assessed socio-demographic data, allergies and cleaning habits. In LAW we also assessed types of cages used, work tasks and protective clothing.

Results MA concentration was higher in home environments of LAW (median (ng mus $\mathrm{m} 1$ )=11.3) than in controls (median $=1.1 ; \mathrm{p}=0.016 ;$ Kruskal-Wallis test). The highest workplace MA concentration was found in the scullery (median $=145,0000.0$ ), followed by the changing rooms (median $=10.2)$ and staffrooms (median $=7.5$ ). MA concentration was higher in homes of LAW who fulfilled cleaning tasks (cleaning of cages, floors, etc.) $(p=0.034)$ and who changed their linen at home less than once a month $(p=0.024)$. MA concentration at home was not associated with duration of mouse contact $(\mathrm{p}=0.909)$ and age of sleeping mattress at home $(p=0.649)$.

Discussion Spreading of MA from workplace to home environment takes place. LAWs who fulfilled cleaning tasks were found to have higher MA concentration at home. Special focus should be given to reduce MA concentration during cleaning in laboratory animal facilities.

\section{C INHALED FINE PARTICLES INDUCE ALLERGIC INFLAMMATION IN THE LUNGS}

\footnotetext{
1,2,3 E Kuroda*, 1,2 KJ Ishii. 'Laboratory of Adjuvant Innovation, Centre for Vaccine and Adjuvant Research (CVAR), National Institutes of Biomedical Innovation, Health and Nutrition, Osaka, Japan; ' 2 Laboratory of Vaccine Science, WPI Immunology Frontier Research Centre (IFReC), Osaka University, Osaka, Japan; ${ }^{3}$ Japan Science and Technology Agency (JST), PRESTO, Japan
}

10.1136/oemed-2018-ICOHabstracts. 145
It is known that inhaled fine particles such as particle pollutions are associated with allergic responses. Many reports have shown that some particulates function as adjuvant to enhance immune responses, however its underlying mechanisms are still unclear. Here, we will show how particulates induce immune responses in the lungs.

We used aluminium salt (alum) and crystalline silica as inflammatory particulate. These inflammatory particulates stimulated alveolar macrophage to release interleukin-1 $\alpha$ (IL$1 \alpha$ ) through cell death. We also observed IL- $1 \alpha$ release in the bronchoalveolar lavage fluid (BALF) after intratracheal instillation (i.t.) of inflammatory particulate alum in vivo. Released IL- $1 \alpha$ in the lungs was thought to be a damage-associated molecular pattern (DAMP) and contributed to antigen-specific IgE production in mouse model of particulate-induced allergic inflammation. In addition, we found the lymphoid cluster formations in the lungs after i.t. alum instillation and allergen exposure. This lymphoid cluster was considered to be inducible bronchus-associated lymphoid tissue (iBALT), and we suggest that iBALT formation might be an important role for local IgE responses in the lungs.

These results indicate that inhalation of particulates and allergen exposure induces and exacerbate allergic responses though the unique immune responses in the lungs such as alveolar macrophage death and iBALT formation.

\section{7d BEEKEEPER ALLERGY RISK AND WORK SUITABILITY}

M Di Gioacchino*, M Di Giampaolo, C Petrarca. Unit of Immunotoxicology and Allergy, CSIMET, G. d'Annunzio University, Chieti, Italy

\subsection{6/oemed-2018-ICOHabstracts.146}

Introduction The risk for a beekeeper to become allergic to bee venom is higher than the general population. The possibility that they develop an allergy is greater when rarely bite with $2 / 3$ month interval. On the contrary, when bites are simultaneous or tight over time the probability of allergy decreases. Beekeepers who receive more than 200 stings do not develop venom allergy. In general, subjects developing a reaction limited to the skin have $70 \%$ possibility of a spontaneous recovery. On the contrary, people haveing a systemic reaction tends to show the same or greater reaction when restung.

Discussion Work suitability of bee allergic beekeepers, which had a systemic reaction is a relevant problem for occupational doctors. They should be prescribed appropriate DPI and provided of auto-injectable adrenaline as emergency treatment, sure that they received the necessary training. Furthermore, it is desirable that every allergic subject receives a desensitising treatment, as it is efficacious in $95 \%$ of patients. The treatment will last for 5 years, except for subjects with high basal tryptase who should be treated for a life-time, as the risk of anaphylaxis reappears after the discontinuation of an already effective immunotherapy. The cardiac involvement during anaphylaxis is very frequent with the picture of Kounis Syndrome (KS), characterised by infarction induced by the effects on coronaries of mast cell chemical mediators. In particular, histamine, usually vasodilator agent, can induce vasoconstriction subjects with coronary endothelial lesions. In this situation, the coronary spasm worsens the perfusion of a tachy heart, already with increased blood demand, with a potentially lifethreatening ischemia. Furthermore, in a subject developing a 